\title{
Polysèmes
}

Revue d'études intertextuelles et intermédiales

\section{La figure de Lilith dans l'œuvre de Dante Gabriel Rossetti : des tableaux aux sonnets (1864-1873)}

\section{Françoise Dupeyron-Lafay}

\section{(2) OpenEdition}

Journals

\section{Édition électronique}

URL : http://journals.openedition.org/polysemes/678

DOI : $10.4000 /$ polysemes. 678

ISSN : 2496-4212

Éditeur

SAIT

\section{Édition imprimée}

Date de publication : 1 janvier 2012

Pagination : 183-202

ISSN : 0999-4203

\section{Référence électronique}

Françoise Dupeyron-Lafay, "La figure de Lilith dans I'œuvre de Dante Gabriel Rossetti : des tableaux aux sonnets (1864-1873) », Polysèmes [En ligne], 12 | 2012, mis en ligne le 01 mars 2015, consulté le 04 mai 2019. URL : http://journals.openedition.org/polysemes/678; DOI : 10.4000/polysemes.678

Ce document a été généré automatiquement le 4 mai 2019.

Polysèmes 


\title{
La figure de Lilith dans l'œuvre de Dante Gabriel Rossetti : des tableaux aux sonnets (1864-1873)
}

\author{
Françoise Dupeyron-Lafay
}

\section{NOTE DE L'AUTEUR}

La date de 1873 correspond à la révision finale du tableau à l'huile intitulé Lady Lilith dont la version initiale est datée de 1864-68.

1 Le cas du tableau intitulé Lady Lilith (1864-1868), et de ses liens avec le sonnet "Lilith " (1866) ${ }^{1}$, est véritablement paradigmatique du fonctionnement de l'œuvre de Rossetti, célèbre pour ses doubles œuvres d'art, des diptyques composés d'un tableau accompagné d'un poème, les deux étant indépendants mais aussi interdépendants. Selon les cas, l'artiste faisait apparaitre le texte de son poème sur le cadre du tableau lui-même, ou sur la toile, ou au dos, ou pas du tout, le poème n'apparaissant que sur papier. Comme on peut le lire dans « The Rossetti Archive: Double Works » :

Rossetti's double works emerge from two established traditions, one textual and one pictorial, that he exploited and developed in important ways. The textual tradition is the "poem on the subject of a picture" [...]. This genre proliferated in the nineteenth century through the wildly popular gift books and annuals, which featured engravings with "illustrative" poems or stories. Rossetti's contributions to this genre include some of his most important works. He called them in general "Sonnets for Pictures", a rubric under which he would eventually gather some of his own double works. ${ }^{2}$

De même que le sonnet qui nous occupe porte deux titres différents (respectivement "Lilith» et «Body's Beauty»), selon son appartenance à la section «Sonnets for Pictures" de Poems (1870) ou à la section «The House of Life. A Sonnet Sequence » de Ballads and Sonnets (1881), le tableau lui-même comporte au moins deux variantes, l'une de 
1864-68 (une œuvre à l'huile) et l'autre, une aquarelle et gouache, dont la datation est problématique $^{3}$ mais estimée être de 1867.

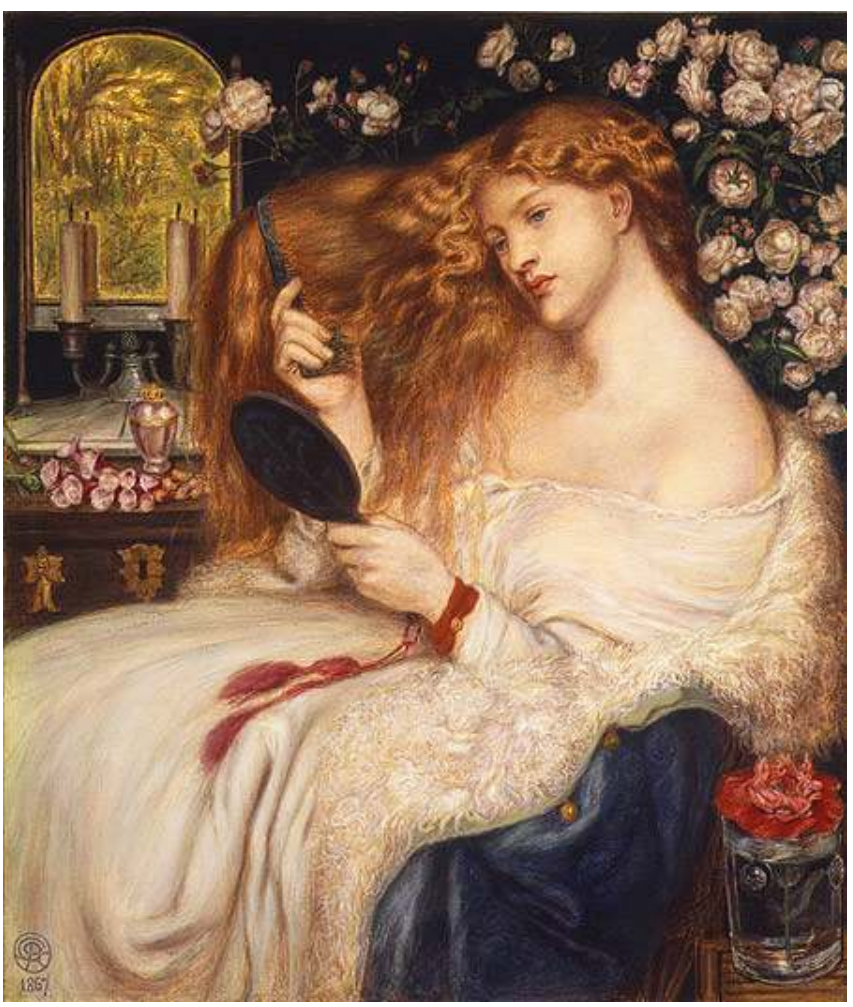

Dante Gabriel Rossetti, Lady Lilith (1867), aquarelle et gouache sur papier, 51,3×44cm, Metropolitan Museum of Art, Creative commons

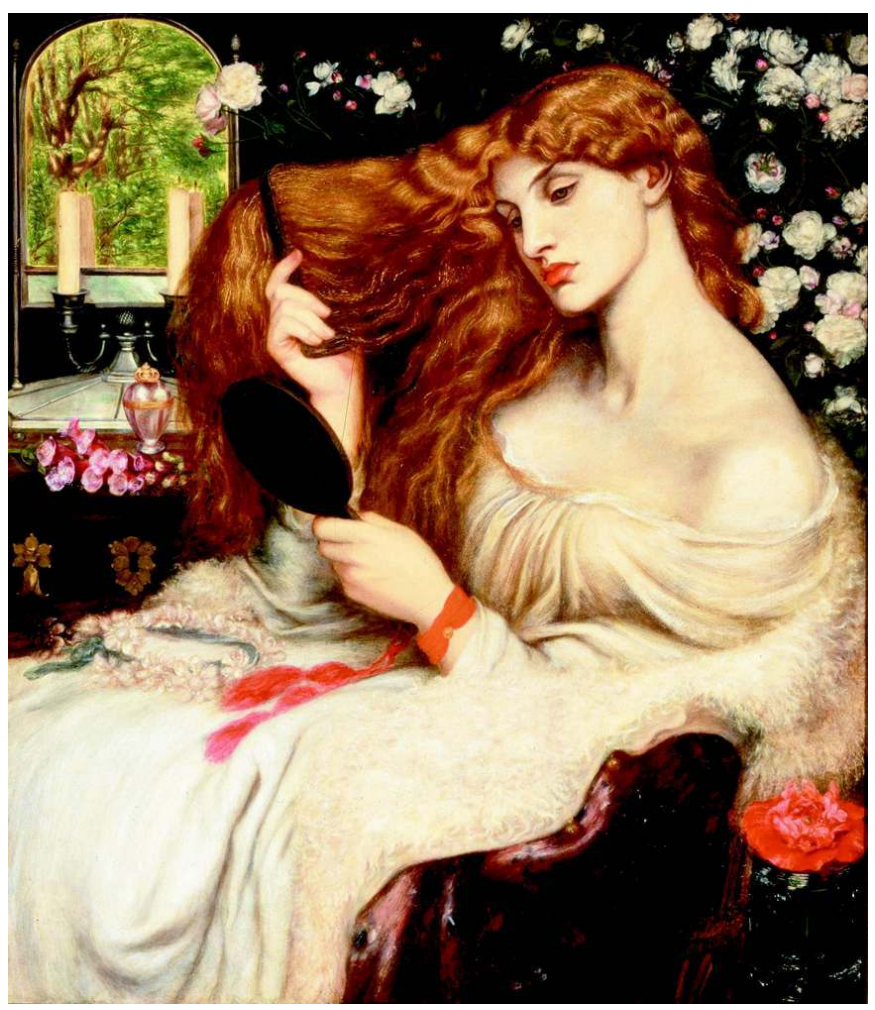

Dante Gabriel Rossetti, Lady Lilith (1864-68, modifiée 1872-73), huile sur toile, 97,8×85,1 cm, Delaware Art Museum, Creative Commons 
On voit bien, en tout état de cause, le processus de transposition d'un medium à l'autre ; d'ailleurs, les deux variantes (image/texte) portent le même titre (c'est généralement le cas chez Rossetti quand il y a ce genre de doublet; on le voit par exemple avec le tableau A Sea Spell (1877) et avec le poème du même titre qui apparaît dans la section « Sonnets " de Ballads and Sonnets et qui est intitulé "A Sea-Spell (for a picture) », tout comme c'est le cas pour « Astarte Syriaca » (tableau de 1877), ou encore «Proserpina » (tableau de 1874) et «La Bella Mano" (tableau de 1875) qui portent tous ce même sous-titre «for a picture ", montrant bien leur vocation d'accompagnement et soulignant la primauté du tableau, chose assez surprenante dans la mesure où, au XIX ${ }^{e}$ siècle, la peinture ne jouissait pas en Angleterre du prestige qui entourait la poésie, et paradoxe aussi, s'il faut en croire le frère de Rossetti, William Michael, dans sa Préface de 1886 aux Complete Poetical Works :

I have not unfrequently heard my brother say that he considered himself more essentially a poet than a painter. To vary the form of expression, he thought that he had mastered the means of embodying poetical conceptions in the verbal and rhythmical vehicle more thoroughly than in form and design, perhaps more thoroughly than in color. ${ }^{4}$

Quoi qu'il en soit, Rossetti inclut dans ses séries appelées "Sonnets for Pictures, and Other Sonnets» (dans le recueil intitulé The Blessed Damozel and Other Poems, appelé communément Poems, à des fins de simplification), «For 'The Wine of Circe,' by Edward Burne Jones", "Venus Verticordia", "Pandora", entre autres, qui correspondent respectivement à des tableaux portant exactement le même titre: Venus Verticordia (1864-68), Pandora (1879).

4 Cette transposition est effectuée par le même artiste au sein de sa propre production, en vase clos en quelque sorte. Néanmoins, si cette transposition est majoritairement «intratextuelle», il faudrait cependant s'interroger brièvement sur l'«intertexte » (mythologique et textuel) étant donné que la peinture de Rossetti est très narrative, d'inspiration très littéraire et que la transposition est donc double au sein de son œuvre: le tableau Lady Lilith illustre tout d'abord un intertexte, puis, il sera lui-même illustré par un texte à l'intérieur du corpus de Rossetti. En " aval », le travail d'échos intertextuels se poursuivra et d'autres versions de Lilith verront le jour, comme celle de Collier, datée de 1887, mais ce qui nous intéressera ici sera l'«amont ", notamment les productions de l'époque romantique comme les deux poèmes de Keats, «Lamia » $(1819-20)^{5}$ et «La Belle Dame sans Merci » (1819-20), un texte lui-même inspiré de la ballade d'Alain Chartier datée de 1424. La Belle Dame du poème, aux cheveux longs, à la voix enchanteresse comme celle des sirènes, asservit les hommes et les conduit à leur perte. Mais c'est surtout un passage du premier Faust (1808) de Goethe qui est non seulement à l'origine de l'entrée de Lilith dans les domaines littéraire et pictural, alors qu'elle relevait jusque-là du mythe religieux, mais qui a aussi façonné ses représentations physiques en mettant l'accent sur le pouvoir magique et fatal de ses cheveux, lieu de sa puissance érotique et instrument mortifère ${ }^{6}$. Ce que les traductions anglaises désignent comme la «Walpurgis Night» (ou Walpurgisnacht), autrement dit la «Nuit de Sabbat» dans la traduction française en prose de Gérard de Nerval, présente donc Lilith pour la première et unique fois. Mephistophélès la décrit à Faust en ces termes : « La première femme d'Adam. Tienstoi en garde contre ses beaux cheveux, parure dont seule elle brille: quand elle peut atteindre un jeune homme, elle ne le laisse pas échapper de si tôt $»^{7}$.

5 Rossetti écrivait généralement ses poèmes après avoir élaboré ses tableaux; le texte venait donc à l'appui de l'image, pour l'illustrer. L'étymologie du verbe illustrer en dit 
long, d'ailleurs, puisque l'illustration a pour vocation d'éclairer, d'illuminer. Chez Rossetti, il s'agit donc d'une forme de paradoxe puisque l'image se voit dotée d'un surcroît de pouvoir (visuel et signifiant) grâce au texte. C'est pourtant l'inverse qui se produit généralement avec l'adjonction de l'image comme illustration du texte. Le degré de hiérarchisation qui régit les relations entre l'illustration et ce qu'elle illustre sont à l'évidence au cœur de cette étude sur la transposition. Et il s'agira de voir qui illustre qui ou s'il y a authentique réciprocité.

On citera cependant une exception notable dans l'ordre chronologique habituel, montrant un changement ponctuel de priorités ${ }^{8}$; le cas de l'image venant prolonger la ballade «Eden Bower» (1869), le poème ayant pour une fois été créé en premier, accompagné d'un croquis en noir et blanc exécuté spécialement pour l'illustrer'9. Autre fait marquant, Lilith y est représentée nue, un serpent enroulé amoureusement autour de son corps $^{10}$. On notera au passage, d'ailleurs, que le serpent (avec ses connotations sexuelles ambiguës, à la fois comme double de Lilith et symbole phallique) et la nudité explicite d'«Eden Bower" sont par ailleurs absents de l'œuvre de Rossetti, et en particulier du tableau Lady Lilith dans lequel l'érotisme est exprimé d'une manière moins directe, comme nous le verrons.

7 Ce phénomène exceptionnel chez Rossetti du visuel composé après le textuel fait évidemment se poser avec acuité la question de savoir quelle relation entretiennent exactement ses tableaux et ses poèmes. Même si, aux dires de son frère, il manquait de confiance en ses dons picturaux qu'il jugeait inférieurs, Rossetti pratiqua pourtant la peinture et la poésie avec un égal talent, hésitant toute sa vie entre ces deux formes d'art. C'est ce qui explique sans doute pourquoi il éprouvait le besoin de créer deux œuvres avec deux supports différents sur le même thème ou le même personnage, pourquoi il lui fallait une deuxième version sur le même sujet. Nous pouvons cependant nous demander s'il y a ajout ou complément, le poème jouant le rôle de post-scriptum (clarification ou explication). Y a-t-il approfondissement et apport réel du poème par rapport au tableau ? Y a-t-il jeu de miroir entre eux: se réfléchissent-ils mutuellement ou se contredisent-ils ? Le reflet mutuel est-il donc fidèle ou peut-on parler d'anamorphose? Étant donné l'ampleur de sa production, je me limiterai aux œuvres directement consacrées au personnage de Lilith, c'est-à-dire le sonnet "Lilith » (ou sa variante « Body's Beauty ») et la ballade «Eden Bower ». Cependant, on peut, ne serait-ce que brièvement, évoquer le fragment inachevé intitulé "The Orchard Pit »11 (1869) qui s'ouvre sur une image de pommier et fonde le pouvoir de séduction de la femme anonyme du poème - une création composite évoquant tout autant Lilith qu'une sirène et la «Belle Dame » de Keats - sur ses cheveux et sa voix enchanteresse.

Ainsi faut-il voir la série tableau-poème que constituent les Lilith comme une forme de diptyque ou bien comme une duplication (mais pas à l'identique) reposant sur une hiérarchisation de type loi de primogéniture avec la considération proportionnelle qui en découle? En d'autres termes, faut-il voir le poème comme une peinture en mots, un prolongement visuel et textuel (ou «iconotextuel ») du tableau lui-même, mais tout de même une œuvre à part entière et non une production subalterne exclusivement au service du tableau et lui servant de paraphrase ? Ou au contraire, le poème, dépourvu de toute autonomie, n'est-il à prendre que comme un prolongement explicatif du tableau, une ekphrasis de Lilith? Ou, troisième option, le poème serait-il en concurrence avec le tableau en ce sens qu'il peut exprimer et expliciter ce qui n'était qu'allusif et implicite, voire invisible et incompréhensible, sur la toile; le poème aurait-il le pouvoir de rendre 
sensible ce que le tableau n'aurait pas suffi à rendre suffisamment vivant et signifiant sans le support des mots ? Rossetti, en écrivant le poème aurait-il cherché, hanté par la figure de Lilith, et insatisfait des résultats, à continuer à "peindre» son tableau? Et d'ailleurs son Lady Lilith lui-même comporte au moins deux versions différentes exécutées avec deux techniques différentes ${ }^{12}$ sur lesquelles Lilith n'a pas les mêmes traits. Le terme de transposition se justifie donc doublement ici puisqu'il s'agit de la signification du mot « métaphore » ou transport, passé du grec au latin, et qu'il s'agit justement d'un mode de fonctionnement privilégié de la poésie.

9 Les transpositions de Rossetti vont générer un mouvement de navette. Le lecteur du poème va tenter d'y retrouver des éléments du tableau ; de plus, même s'il ne connaît pas le poème qui fait suite au tableau, le lecteur-spectateur va tenter de lire un texte dans ce même tableau, notamment en y superposant un intertexte très composite, mi-littéraire, mi-visuel, qui lui est familier, une sorte de palimpseste «iconotextuel» couvrant plusieurs décennies, voire des siècles : la tradition juive médiévale entourant Lilith, les récits mythologiques mettant en scène les sirènes, Goethe et Keats, la symbolique chrétienne de la vanité et la signification du miroir ${ }^{13}$, du peigne et des cheveux comme signe de luxure et de péché, partie du corps véritablement fétichisée à forte charge érotique, redoutable instrument de séduction comme on le voit bien aussi dans d'autres tableaux de l'époque, préraphaélites ou non, comme celui de Lord Leighton, The Fisherman and the Siren (1858). L'association étroite entre la chevelure, la séduction et l'érotisme mortifère est aussi l'un des motifs centraux du roman de Mary Elizabeth Braddon, Lady Audley's Secret (1862), dans lequel les cheveux de Lucy Audley donnent lieu à une comparaison avec la sirène, personnage de femme fatale d'ailleurs très proche de celui de Lilith ${ }^{14}$. Rossetti avait d'ailleurs écrit le sonnet «A Sea-Spell »15 (1868) qui, comme «The Orchard Pit " mêlait les caractéristiques attribuées à la sirène (le chant irrésistible et mortel) et celles de Lilith (le pommier et l'image des rets fatals): «Her lute hangs shadowed in the apple-tree, / While flashing fingers weave the sweet-strung spell ( (1l. 1-2). Dans Texte/Image. Images à lire, textes à voir, Liliane Louvel se penche sur cette " oscillation de la lecture que suscitent les aller-retour incessants de l'image au texte ", évoquant le «mouvement perpétuel entre voir-lire, d'où la production de ces ondes du visible qui n'en finissent pas de trembler à la surface du lisible » et les "interférences provoquées par le dynamisme inhérent à l'iconotexte $»^{16}$. Il y a système de dialogue, de traduction, d'interprétation, de transport (on retrouve donc la métaphore) mais du fait du passage perpétuel entre deux codes sémiotiques, le "lecteur" n'est "jamais totalement dans l'un, ni totalement hors de l'autre $»^{17}$. Cela nécessite une « démarche de transaction, de négociation $\aleph^{18}$, une lecture active et dynamique. Comment le détail visuel sera-t-il transcrit dans le poème et quels seront les mécanismes de la métaphore?

10 Je tenterai d'abord de décrire le tableau, Lady Lilith. Le personnage de Lilith y est sécularisé, ou du moins dégagé de son contexte biblique ou mythologique traditionnel, comme en témoigne l'adjonction de Lady au titre, transformant Lilith en personnage victorien. La femme qui y est représentée ressemble à bien des héroïnes de la peinture de Rossetti avec sa longue, épaisse et flamboyante chevelure aux teintes blond roux et aux ondulations serpentines qui est le point focal du tableau ; elle s'inscrit par ailleurs dans un intérieur assez intemporel (très difficile à dater précisément en tout état de cause) mais qui n'a en tout cas rien d'antique et ressemble à un boudoir. Le cadre présente cependant une ambiguïté radicale dans la mesure où le miroir placé à l'arrière-plan ressemble à une fenêtre mais reflète les deux bougies placées devant ainsi qu'un décor 
boisé extrêmement luxuriant (rappel du jardin d'Éden?), que complètent les fleurs qui environnent Lilith, de sorte que l'on ne sait si ce «boudoir " est une pièce close ou se situe à l'extérieur ${ }^{19}$. La femme, de trois-quarts, semble uniquement préoccupée d'ellemême, de son apparence, de sa beauté qu'elle contemple dans un miroir qu'elle tient de la main droite tout en se peignant ; c'est le « And, subtly of herself contemplative » du vers 6 du sonnet ${ }^{20}$. Lilith est la seule à voir cette image reflétée dans le miroir dont le « lecteur ", pour sa part, ne voit que le dos de couleur sombre, qui évoque un trou noir au cœur du tableau, part d'ombre et de mystère absolue et inquiétante.

11 De plus, contrairement à la Lilith nue et svelte illustrant la ballade "Eden Bower », la Lilith du tableau (qui préexiste à celle du sonnet contrairement à ce qui se passe pour "Eden Bower ", rappelons-le) est partiellement vêtue et il faut donc que sa sensualité se traduise autrement. On remarque que non seulement sa gorge et son épaule gauche sont découvertes, mais que, d'autre part, elle ne porte visiblement pas de corset. Cette absence, ainsi que ses cheveux lâchés, en disent long sur sa sexualité. Sa tenue, fluide comme un déshabillé, laisse donc plus que deviner ses formes généreuses, notamment sa poitrine opulente. Le fait aussi que cette Lilith soit nonchalamment assise sur une fourrure laisse deviner sa sensualité et sa part d'animalité. La blancheur aux teintes ivoire du vêtement et de la fourrure font écho à celle de la chair mais une fleur rouge en bas et à droite du tableau (s'agit-il d'une rose ou d'un gros pavot? Il s'agit en tout cas de la seule, toutes les autres, plus petites, situées dans la partie supérieure du tableau, étant rose pâle ou blanches ${ }^{21}$, symboles de l'amour stérile), ainsi que le ruban rouge accroché au poignet gauche de Lilith évoquent le sang, le plaisir, et la mort. Tout " détail », au sens de partie constitutive de l'ensemble et non au sens d'accessoire sans importance, naturellement, est éminemment signifiant et constitue un texte à lui seul, un «iconotexte ", donc, qui entre en résonance étroite avec les mots du sonnet. En peinture, le détail visuel est potentiellement texte et métaphore et cela se confirme bien avec les digitales (placées sous le miroir), symboles d'inconstance, avec la bouche rouge et charnue de Lilith, et avec la fleur rouge.

12 C'est clairement le cas aussi du ruban et des cheveux, figuration oblique et pourtant «parlante » du topos des liens et de la captivité : ils sont comme des chaînes asservissant les hommes amoureux de Lilith, et les conduisant à leur perte. Le sonnet les décrit comme de l'or et le vers 4 souligne leur danger en évoquant indirectement le goût du lucre: "And her enchanted hair was the first gold». Le cheveu métaphorise le piège exprimé dans le sonnet par l'expression "her enchanted hair » (vers 4$)^{22}$. La chevelure, par un procédé stylistique qui évoque l'univers de la littérature fantastique, est à la fois objet et sujet, inanimé et animé, « enchanted » et implicitement « enchanting ». Le poème, quand il s'agit d'évoquer l'asservissement des hommes par Lilith, est cependant moins allusif avec son réseau lexical insistant : « the bright net she can weave » (vers 7) ${ }^{23}$, « Till heart and body and life are in its hold» (vers 8), « snare » (vers 11), « and left his straight neck bent » (vers 13), image de la résistance domptée et brisée.

13 C'est peut-être bien en cela que réside ce «reste » qu'évoque Liliane Louvel ${ }^{24}$, puisque, paradoxalement, c'est le surplus d'explicite qui enlève sa profondeur stratifiée ou sédimentaire au poème en le faisant fonctionner selon un axe de prime abord exclusivement horizontal, par rapport à lui-même et en le privant du fonctionnement de type palimpseste (selon un axe vertical, donc) qui caractérise justement le tableau. Le simple fait de regarder le visible en fait une histoire lisible et déclenche chez le 
spectateur, à partir de détails visuels disséminés sur la toile, un processus simultané de lecture de textes multiples superposés en couches invisibles.

Mon propos n'est évidemment pas, loin s'en faut, de dévaloriser le poème qui possède une qualité intrinsèque et n'aurait pas du tout été envisagé de la même manière si le tableau n'avait pas existé. C'est juste qu'il raconte, à première vue, l'histoire de Lilith d'une façon plus discursive et narrative tandis que le tableau le fait d'une façon plus multidirectionnelle mais synchronique, dans la mesure où le spectateur averti (connaissant les codes picturaux et le contexte littéraire et pictural victorien) convoque quasi instantanément l'intertexte et où sa lecture et son interprétation du détail signifiant sont presque simultanées - ou alors, elles n'ont pas lieu. La lecture est donc moins aidée, moins dirigée, moins orientée linéairement que celle du poème. Elle peut être aussi plus vagabonde...

15 Mais elle est peut-être plus lacunaire aussi car, d'un autre côté, le poème clarifie ce qui n'apparaît peut-être pas aussi nettement dans le tableau du fait de sa trop grande richesse esthétique, de la trop grande beauté du modèle dont seuls les yeux (et peut-être la bouche ?) pourraient, si on les regarde bien, traduire l'impitoyable cruauté, moins évidente encore dans l'aquarelle ${ }^{25}$ de 1867. Les méfaits de la femme fatale, racontés dans le sonnet, ne sont que sous-entendus sur le mode visuel dans le tableau où Lilith est au repos, immobile, exception faite de sa main droite qui soulève sa lourde chevelure. La mort est seulement suggérée dans Lady Lilith mais le ruban rouge tout en évoquant les liens, la captivité et la couleur du sang, ou la chevelure tout en convoquant une longue tradition diabolisant l'érotisme féminin dont elle est le symbole par excellence, ou encore la peau de bête, tout en rappelant la férocité du fauve ou la cruauté du félin, ne font pas nettement présager la violence infligée et la mort, chose que les deux derniers vers du sonnet mettent en scène sur le mode métonymique (synecdoques notamment): la «strangulation du cœur » de l'amant par un « cheveu d'or » de Lilith (vers 14), la brûlure de ses yeux (vers 12), la blessure du flanc ou de la poitrine transpercée ("through », vers 14), la décollation potentielle car « his straight neck bent » (vers 14) annonce l'image du condamné à mort s'apprêtant à poser sa tête sur le billot... Cet amant meurtri, torturé et pris au piège demeure invisible dans le tableau et peut-être le spectateur (masculin?), fasciné, est-il censé en partie jouer ce rôle?

Il faut aussi signaler que la Lilith du tableau perd une partie de sa dimension mythique car Adam et le serpent en sont à l'évidence totalement absents. L'aura séculaire de Lilith, éternellement jeune, est célébrée dans le sonnet («And still she sits, young while the earth is old", vers 5) et le serpent y est non seulement cité mais incarné, pourrait-on dire, par le jeu des allitérations obsédantes (fricatives sifflantes et chuintantes) des vers 10 et 11 qui miment le jeu de la séduction et rappellent sa parenté étroite avec Lilith: "That, ere the snake's, her sweet tongue could deceive» (vers 3) ${ }^{26}$. Malgré les connotations inquiétantes qui l'entourent, la Lilith du tableau n'apparaît pas non plus sous les traits d'une sorcière : « (The witch he loved before the gift of Eve) » (vers 2), « her enchanted hair » (vers 4), «Thy spell» (vers 13). De plus, si la rose est (sur)représentée dans le tableau, le coquelicot (ou pavot) n'y apparait pas très clairement (s'agit-il de la fleur rouge déjà évoquée, placée en bas et à droite ?) alors qu'il est mentionné au vers 9 $\mathrm{du}$ sonnet: "The rose and poppy are her flower...». Le rappel le plus évident du coquelicot dans le tableau est donc la couleur rouge ; il s'agit pourtant d'un symbole floral important, à la fois emblème de mort mais aussi représentation de l'oubli bienheureux. La très belle et très synthétique métaphore du vers 11 , « soft-shed kisses » concentre en fait 
plusieurs motifs apparentés, celui des larmes ou du sang versés (image de souffrance et de mort), des pétales de fleur ou des feuilles qui se fanent et tombent (image de stérilité et de mort) et l'on s'aperçoit que cette métaphore constitue de surcroît un oxymore puisque le baiser d'amour est celui qui donne la mort. Il s'agit de l'essence même du pouvoir de Lilith.

À y regarder de plus près, le poème est finalement susceptible de se lire plus verticalement qu'il n'y paraissait mais son jeu de résonances est plus d'ordre lexical et sonore qu'intertextuel (bien que placé à l'ombre de Goethe et de Keats) et le palimpseste littéraire et pictural y est bien plus discret que dans le tableau.

On retrouve en fait la définition de la métaphore que donne Gérard Genette dans Figures $I$ : «Ainsi la métaphore n'est pas un ornement, mais l'instrument nécessaire à une restitution, par le style, de la vision des essences, parce qu'elle est l'équivalent stylistique de la mémoire involontaire, qui seule permet, en rapprochant deux sensations séparées dans le temps, de dégager leur essence commune par le miracle d'une analogie [...] $\aleph^{27}$. La métaphore, comme l'allusion intertextuelle en peinture, transforme la diachronie en synchronie (en spatialisant ce qui était de l'ordre du temporel, en court-circuitant la chronologie) et, dans le cas qui nous occupe, contribue aussi l'espace d'un instant à abolir le fossé méthodologique apparent qui séparait le tableau et le poème.

Le sonnet, quoique comportant des éléments à forte charge visuelle comme l'or de la chevelure, les regards captivés, le tissage des rets de l'enchanteresse, les fleurs, n'a donc rien, on le voit au terme de cette analyse comparée, d'une simple ekphrasis; il raconte à la vérité une tout autre histoire que le tableau, plus proche en cela de la tradition religieuse apocryphe (par rapport aux textes religieux de la stricte orthodoxie juive) dans laquelle s'inscrit Lilith $^{28}$. Par ailleurs, le poème convoque une troisième forme d'art qui est la musique, ce chant de Lilith, doux murmure, mortel comme celui des sirènes, qui s'égrène au fil du sonnet. On pourrait dire, en somme, qu'il y a «reste» et déperdition, une inévitable part d'ombre et de silence qui demeure au «bout du compte " (pour reprendre l'image financière de Liliane Louvel), tant dans le tableau que dans le sonnet; mais à mon sens, même si Rossetti a conçu son poème comme une «illustration ", et le sous-titre de sonnet «for a picture » le montre bien, il me semble que ce poème a l'immense mérite de recadrer le tableau, de le recentrer et de l'enrichir, de lui donner une plus grande profondeur de champ, notamment en le recontextualisant par rapport à la tradition historique et mythologique ${ }^{29}$. Dans le sonnet, Lilith est une figure intemporelle, aussi «vraie» dans l'Antiquité qu'à l'époque victorienne, une représentation de la part maudite de l'éternel féminin et non pas seulement l'expression de préoccupations d'ordre personnel, intime, autobiographique, impression que donne la peinture de Rossetti. D'ailleurs le vers 1 débute en ces termes: «Of Adam's wife, Lilith, it is told» (je souligne), ce qui confère au poème sa dimension légendaire et fait du poète le conteur d'un vieux mythe ${ }^{30}$.

Ainsi, après avoir lu le sonnet, on ne peut qu'apprécier davantage ce magnifique tableau qui, sans le poème, en serait peut-être resté au stade d'une représentation parmi d'autres d'une femme fatale parmi d'autres, les détracteurs de Rossetti critiquant d'ailleurs les grandes similitudes entre les héroïnes de ses tableaux, chose peu surprenante en fait, puisque Rossetti utilisa toute sa vie les mêmes modèles, symboles pour lui de la beauté idéale, qu'elle soit du corps ou de l'âme : Elizabeth Siddal (sa femme, épousée en 1860 et morte en 1862), Fanny Cornforth, Jane Morris (femme de William Morris), Alexa Wilding, Annie Miller. 
21 La Lilith du tableau a le visage d'une Victorienne bien réelle et permet d'exprimer ou de donner corps à la peur mêlée de fascination qu'inspirait le féminin à Rossetti et à ses contemporains. Cette Lady Lilith, tout en étant une œuvre d'art réussie, ressemble presque à un symptôme et elle a en tout cas valeur de témoignage ou de confession d'ordre quasi autobiographique. La Lilith du sonnet, au contraire, est sans visage et relève plus de l'imaginaire collectif. Ainsi, sans aucun doute, tels les deux volets d'un diptyque, le tableau et le sonnet s'illustrent et s'enrichissent mutuellement, formant une œuvre d'art complète par cette association si fertile, et offrant donc au lecteur-spectateur une expérience esthétique totale.

\section{BIBLIOGRAPHIE}

\section{Sources primaires}

Braddon, M.E. Lady Audley's Secret (1862), Oxford: Oxford World's Classics, 1998.

Buckler, William E. (ed.). Walter Pater: Three Major Texts. New York and London: New York UP, 1986.

Rossetti, William M. The Complete Poetical Works of Dante Gabriel Rossetti. Honolulu, Hawaii: UP of the Pacific, 2001 ( $1^{\text {st }}$ pub. 1901 edition).

“The Rossetti Archive" sur www.iath.virginia.edu/rossetti ou http://www.rossettiarchive.org

\section{Sources secondaires}

Edwards, Meghan. "The Devouring Woman and her Serpentine Hair in Late-PreRaphaelitism": www.victorianweb.org/painting/prb/edwards12.html

Genette, Gérard. Figures I. Paris : Minuit, 1966.

Louvel, Liliane. Texte/Image. Images à lire, textes à voir. Rennes : PUR, collection « Interférences ", 2002.

\section{NOTES}

1. «Lilith» (1868) fut initialement publié dans la revue-pamphlet de Swinburne Notes on the Royal Academy Exhibition, puis en 1870 dans la section « Sonnets for Pictures » de Poems. Ensuite, dans le but d'établir un contraste entre la beauté charnelle et la beauté spirituelle, une polarité récurrente dans son œuvre, Rossetti, en 1881, décida d'intégrer son poème au recueil Ballads and Sonnets, dans la section "The House of Life. A Sonnet Sequence" (dans sa deuxième partie, « Change and Fate »), et il en modifia le titre qui devint « Body's Beauty (sonnet 78) pour former un diptyque avec "Soul's Beauty» (sonnet 77), ces deux poèmes accompagnant respectivement les tableaux Lady Lilith et Sibylla Palmifera (1866-70). Le passage d'un recueil à l'autre n'entraîne qu'une seule modification textuelle: "watch the bright net she can weave", au vers 7 de «Lilith», devient « watch the bright web she can weave» au vers 7 de «Body's Beauty». Les 
deux recueils The Blessed Damozel and Other Poems, et Ballads and Sonnets dont sont tirés les poèmes à l'étude dans le présent article, sont réunis dans l'édition de référence suivante, éditée et préfacée par le frère du poète, The Complete Poetical Works of Dante Gabriel Rossetti, William M. Rossetti (ed.), Honolulu, Hawaii: UP of the Pacific, 2001 (reprinted from the 1901 edition).

2. On peut consulter «The Rossetti Archive » sur www.iath.virginia.edu/rossetti (cette adresse est aussi accessible par http://www.rossettiarchive.org).

3. On peut consulter le site www.metmuseum.org à ce sujet. Alexa Wilding semble avoir prêté ses traits au tableau à l'huile que nous connaissons mieux que l'aquarelle de 1867 (?) sur laquelle Lilith ressemble plutôt à Fanny Cornforth. Il s'agit de deux modèles habituels de Rossetti, outre sa femme Elizabeth Siddal, morte en 1862, et Jane Morris (née Burden).

4. W.M. Rossetti, The Complete Poetical Works, op. cit. (voir note 2), xxvi.

5. On relève un écho direct dans les deux tableaux de John William Waterhouse, deux Lamia, datées respectivement de 1905 et de 1909.

6. C'est précisément le bref passage consacré à Lilith dans le Faust de 1808 de Goethe qui sert de légende (inscrite au dos de l'œuvre) à l'aquarelle Lady Lilith (1867) de Rossetti qui connaissait le texte à travers la traduction qu'en avait faite Shelley, puis qui en proposa sa propre traduction : « Hold then thy heart against her shining hair, / If, by thy fate, she spread it once for thee, / For, when she nets a young man in that snare / So twines she him he never may be free ". Voir à ce sujet "The Devouring Woman and her Serpentine Hair in Late-PreRaphaelitism" de Meghan Edwards, dans www.victorianweb.org/painting/prb/edwards12.html. On notera que le terme «net » que Rossetti emploie dans sa traduction figurera dans le sonnet «Lilith» de Poems, mais qu'il disparaîtra, comme nous l'avons vu, au profit de « web » dans « Body's Beauty ».

7. Faust (1808), traduit par Gérard de Nerval, Paris : Flammarion / Librio, 1996, 134.

8. Le cas du tableau The Blessed Damozel (1875-78) constitue une deuxième exception, l'écrit précédant l'image du même titre, comme pour « Eden Bower », les deux textes figurant d'ailleurs dans Poems.

9. La Lilith (1887) de Collier, sans doute influencée par celles de Rossetti, s'apparente plus à l'illustration graphique de la ballade « Eden Bower » qu'à la Lady Lilith de 1864-68, en raison de sa nudité et des connotations érotiques du serpent. Il en va de même pour la Lilith (1892) de l'Américain Kenyon Cox.

10. La ballade «Eden Bower » fait du serpent à la fois le double et l'amant de Lilith : «A snake was I when thou wast my lover » (vers 9) ; « O my love, come nearer to Lilith! / In thy sweet folds bind me and bend me, / And let me feel the shape thou shalt lend me! / In thy shape I'll go back to Eden » (vers 67-70) ; « Wreathe thy neck with my hair's bright tether, / And wear my gold and thy gold together! » (vers 104-105), ou encore « How shall we mingle our love's caresses, / I in thy coils, and thou in my tresses! » (vers 113-114).

11. Pour trouver le texte du poème (qui ne figure pas dans The Complete Poetical Works of Dante Gabriel Rossetti, op. cit. en note 1), consulter le site http://eserver.org/feminism/lilith/ femmeftl.html.

12. Voir note 3.

13. On peut penser au tableau de John William Waterhouse intitulé Vanity (1910) et montrant une belle femme aux cheveux roux, attachés en chignon et qu'elle est, semble-t-il, en train de parer d'une rose, décolletée et vêtue de blanc, se regardant dans un miroir avec une évidente complaisance. La juxtaposition de ce tableau et de la Lady Lilith de Rossetti est éclairante. Cependant, le personnage de Waterhouse n'a pas les cheveux lâchés, ce qui réduit considérablement ses connotations négatives.

14. «Lady Audley flitted from room to room [...] now sitting down to the piano to trill out a ballad [...] now strolling into the dressing room to [...] have her curls re-arranged for the third or fourth time [...] ». M.E. Braddon, Lady Audley's Secret (1862), Oxford: Oxford World's Classics, 1998, 77. Plus tard, Robert Audley fait d'elle un rêve très révélateur : «The sleeper saw a pale, starry 
face looking out of the silvery foam and knew it was my lady, transformed into a mermaid, beckoning his uncle to destruction » (246).

15. Il est assez curieux de constater que ce sonnet, composé en 1868, et précédant donc le tableau du même titre, daté de 1877 , est pourtant présenté comme ayant été composé «for a picture » dans Ballads and Sonnets, de 1881. Le tableau existait-il déjà en 1868, même sous forme inachevée ou comme étude préparatoire ? Ou cela signifie-t-il que Rossetti considérait son poème comme subalterne à l'image?

16. Liliane Louvel, Texte/Image. Images à lire, textes à voir, Rennes: PUR, collection «Interférences », 2002, 147.

17. Ibid., 148.

18. Ibid., 149.

19. Le boudoir est l'une des traductions possibles du mot « bower » qui désigne aussi une tonnelle dans un jardin, celui d'Éden, présent dans la ballade «Eden Bower », et sans doute aussi dans le miroir du tableau.

20. On pense aussi au vers 9 de « A Sea-Spell » : « She sinks into her spell [...] ».

21. Une couronne de fleurs blanches, image de virginité et symbole nuptial détournés, est posée sur les genoux de Lilith, et l'on pense aux deux premiers vers de la cinquième strophe de « La Belle Dame sans Merci »: "I made a Garland for her head, / And bracelets too, and fragrant Zone ".

22. Lilith décrira de la même façon son pouvoir sur Adam dans la ballade « Eden Bower » (1869): « All the threads of my hair are golden, / And there in a net his heart was holden » (vers 17-18).

23. Il s'agit du sonnet «Lilith», la version initiale, puisque le vers comporte le mot «net » et non pas « web » comme dans « Body's Beauty ».

24. Il s'agit des conséquences du passage de l'image au texte et du mouvement de va-et-vient que cela nécessite: "comme le change opéré entre deux monnaies, il y a toujours un reste, une différence de valeur dont quelqu'un paie le prix.» (149). La question est évidemment de savoir pourquoi et comment il y a déperdition ou «différence de valeur »? En quoi consiste ce "reste » et qui « paie ce prix » ? Le lecteur-spectateur, l'œuvre, ou les deux?

25. Dans son texte The Renaissance (1873), Walter Pater évoque Léonard de Vinci, et notamment son type de beauté féminine (The Renaissance: Studies in Art and Poetry, "Leonardo da Vinci ", 149-150, Walter Pater: Three Major Texts, William E. Buckler (ed.), New York and London: New York UP, 1986, ma traduction). Les femmes androgynes de sa peinture n'appartiennent pas « à la famille chrétienne, ou à celle de Raphaël». Il s'agit de medium qui, à la façon de "délicats instruments ", nous donnent conscience des « forces de la nature » et de son " magnétisme ». Le «sourire insondable» de la Joconde, idéal féminin de Leonardo selon Pater, a quelque chose de " sinistre », et les yeux aux paupières lourdes, un peu lasses, expriment « d'étranges pensées, des rêveries fantastiques, et des passions extrêmes ». Il s'agit aussi d'une forme de beauté façonnée par l'âme et «toutes ses maladies ». La Gioconda conjugue « l'animalité de la Grèce, la luxure de Rome, le mysticisme médiéval avec ses ambitions spirituelles et ses amours platoniques, le retour du paganisme, et les crimes des Borgia. Elle est plus vieille que les rochers parmi lesquels elle pose; comme le vampire, elle est morte plusieurs fois et a découvert les secrets de la tombe »; elle est à la fois Léda, mère d'Hélène de Troie, et Sainte Anne, mère de Marie.

26. On notera d'ailleurs la fluidité extrême des sonorités du sonnet où abondent les fricatives en [v] et les semi-voyelles [w].

27. Gérard Genette, Figures I, Paris : Éditions de Minuit, 1966, 39-40.

28. L'identité et l'existence de Lilith ne sont attestées que par une certaine tradition biblique occulte. Dans la Bible, Lilith n'apparaît nommément que dans le Livre du prophète Isaïe $(34,14)$ dans «Les ruines d'Edôm », conformément à la tradition mythologique babylonienne. Mais la Genèse ne la présente pas clairement et nommément comme la première épouse d'Adam. Apparemment, les premières références récurrentes et explicites à Lilith en tant que première 
épouse d'Adam datent du Moyen Âge et s'inscrivent dans la tradition occulte de la Kabbale. Lilith ne serait donc qu'une midrash, ou légende d'origine rabbinique, et elle figure abondamment dans le Zohar, texte majeur de la Kabbale apparu au XIII ${ }^{\mathrm{e}}$ siècle et attribué à Moïse de Leon (qui serait lui-même inspiré de L'Alphabet de Ben-Sira, ouvrage médiéval profane et anonyme) qui fait clairement d'elle la première femme d'Adam même si une autre tradition la considère comme l'épouse de Samaël, roi des démons. Raphaël Patai se penche entre autres sur Lilith dans Gates to the Old City. A Book of Jewish Legends, New York: Avon Books, 1980. Les pages 453-469 de son livre (chapitre 5 « Kabbala ») sont consacrées à « The Legend of Lilith » et comportent des citations du Zohar également consultables sur le site: http://ccat.sas.upenn.edu/humm/Topics/Lilith/ origin.html.

29. La ballade "Eden Bower", que l'on peut voir comme une suite et un approfondissement du sonnet, ira plus loin encore dans cette direction en donnant la parole à Lilith et en transformant donc radicalement son image. Comme dans «Lamia » de Keats, Lilith dans « Eden Bower » n'est pas stigmatisée comme un être foncièrement mauvais et le lecteur est invité à compatir dans les deux cas. Comme on peut le lire sur eserver.org/feminism/lilith/edenb.html : « [...] Lilith is here given nearly exclusive speaking space. No longer is her story being told by a biased third person, as in the Zohar [...] [Lilith] tells the story from her own perspective ». Par ailleurs, étant donné qu'elle se présente comme la victime de Dieu et d'Adam, à présent heureux aux côtés d'Ève, et cherche à se venger en demandant au serpent son aide, le lecteur se voit contraint de repenser la Chute et de l'envisager différemment, notamment en raison de l'ambivalence morale de Lilith.

30. Cependant le changement de titre, le passage de «Lilith" à "Body's Beauty " infléchit considérablement le sens de Lilith, initialement personnage mythique mais transformée ensuite en abstraction et en allégorie (en particulier à cause de l'opposition avec « Soul's Beauty »).

INDEX

oeuvrecitee Body's Beauty, Eden Bower, Lady Lilith, Lilith 